\title{
Pulmonary infections
}

\author{
Pedro Daltro • Eloá N. Santos • Taísa D. Gasparetto • \\ Maria E. Ucar • Edson Marchiori
}

Received: 4 January 2011 /Accepted: 31 January 2011

(C) Springer-Verlag 2011
Keywords Pulmonary infection · High-resolution CT . Children

\section{Introduction}

Pneumonia is defined as an acute or chronic disease that causes inflammation of the lungs. It is one of the most common infections in the pediatric population and a leading cause of mortality in children in developing countries. It is usually acquired through inhalation of infected upper airway secretions, although hematogenous routes or contiguity can occur [1-4]. Viruses, bacteria, fungi and other microorganisms are the main etiological agents. Though pneumonia from any organism can occur at any age, certain age groups are at higher risk for particular types of pneumonia. The vast majority of pneumonia in infancy and early childhood is of viral origin and accounts for $50 \%$ of pneumonia in children younger than 5 years [2]. The most common bacterial organism in children older than 5 years is $S$. pneumoniae. Mycoplasma is the most frequent cause of pneumonia in those older than 5 years of age group $[2,4,5]$. The immunological status of the child and the infectious agent also have an important role in the clinical and radiological aspects of the disease $[5,6]$. This paper reviews the most common causes of pulmonary infection in children, emphasizing the imaging findings.

\section{Viral pneumonia}

Viral infections occur after the inhalation of infected aerosols. Usually the infection begins in the nasopharynx and upper respiratory tract and migrates to the small airways and alveoli. The clinical presentation of viral pneumonia varies greatly depending on the infecting agent, 
the age and the immune status of the host $[4,5]$. Influenza virus, respiratory syncytial virus (RSV), adenovirus and parainfluenza are common causes of viral pneumonia in children. In premature-born infants with lung disease, RSV can be devastating. Recently, human metapneumovirus has been recognized as a pathogen. Its ubiquity suggests that it is as important as RSV, and studies suggest its incidence is as high as that of RSV. Nearly $100 \%$ of children ages 5-10 years are seropositive [7]. Herpes simplex virus is rare, affecting mostly newborns. Patients with immunodeficiency syndromes are also at risk of pneumonia caused by other pathogens such as cytomegalovirus [5]. Influenza virus and RSV infect the pneumocytes, causing diffuse alveolar damage. The alveoli are infiltrated with neutrophils, and mononuclear cells with fibrin and edematous fluid $[1,4]$. In addition, the submucosa presents with hyperemia with focal hemorrhage and edema. The small bronchial diameters and poorly developed airways of the smallest children combined with their abundant mucus production cause the characteristic abnormalities revealed by chest radiograph $[4,5]$. CT is sometimes indicated in complicated cases of diffuse disease [2].

The most common chest radiographic findings are bilateral symmetrical parahilar and bronchial opacities with or without atelectasis and air trapping (Figs. 1 and 2). Focal and asymmetrical disease are not uncommon. Lymph node enlargement can occur, but pleural effusion is rare.

Recently viral infection has been recognized as a cause of severe pneumonias leading to respiratory failure and death. Higher mortality rates are associated with coronavirus A (SARS, severe acute respiratory syndrome), and influenza virus type A H5N1 (bird flu) and type A H1N1. As with other viral infections, focal or diffuse interstitial opacities are the initial chest radiograph presentation, but they can progress rapidly to bilateral areas of consolidation (Fig. 3). In children with type A H1N1 initial radiographs were often normal, and a trend to more severe disease was seen in children with diffuse ground-glass opacities [2].
Early recognition of the epidemic and an increased index of suspicion in patients with asthma and chronic lung diseases will help in identifying these pneumonias.

\section{Complications of viral pneumonia}

The most common complication of viral pneumonia is a secondary bacterial pneumonia. Damage to the respiratory tract mucosa harms local defense mechanisms and leaves the lung vulnerable to bacterial superinfection $[1,4,6]$.

Postinfectious bronchiolitis obliterans (BO) is a chronic obstructive disease of the lower airways that affects mainly male infants after an episode of acute viral bronchiolitis. Adenovirus is the most common agent. The virus infects the epithelial cell surface and initiates intracellular replication, resulting in squamous metaplasia of the bronchial mucosa and necrosis of the bronchiolar wall with destruction of the ciliated epithelium. Peribronchiolar inflammatory opacities, edema of the submucosa and connective tissue, and increased mucus secretion cause obstruction of the bronchioles, resulting in air trapping and atelectasis. CT performed in children because of progressive disease shows mosaic perfusion, peribronchial thickening, atelectasis, bronchiectasis, air trapping and sometimes lung volume reduction (Fig. 4) $[8,9]$.

Swyer-James-MacLeod syndrome is a postinfectious BO with characteristic radiological features that are virtually pathognomonic. Typically involvement is unilateral, affecting the entire lung, lobe or segment. The changes observed on chest radiography are hyperlucency, mainly because of pulmonary hypoperfusion; reduction of vascular and hilar markings; volume reduction of the affected lung or lobe, and air trapping on radiographs made at expiration. CT demonstrates these features with more detail and might demonstrate bronchiectasis (Fig. 5).

Bronchiolitis obliterans organizing pneumonia (BOOP) is now called cryptogenic organizing pneumonia and
Fig. 1 Viral pneumonia in a 13month-old boy. Frontal (a) and lateral (b) chest radiographs show pulmonary hyperinsufflation with bilateral and symmetrical parahilar opacities
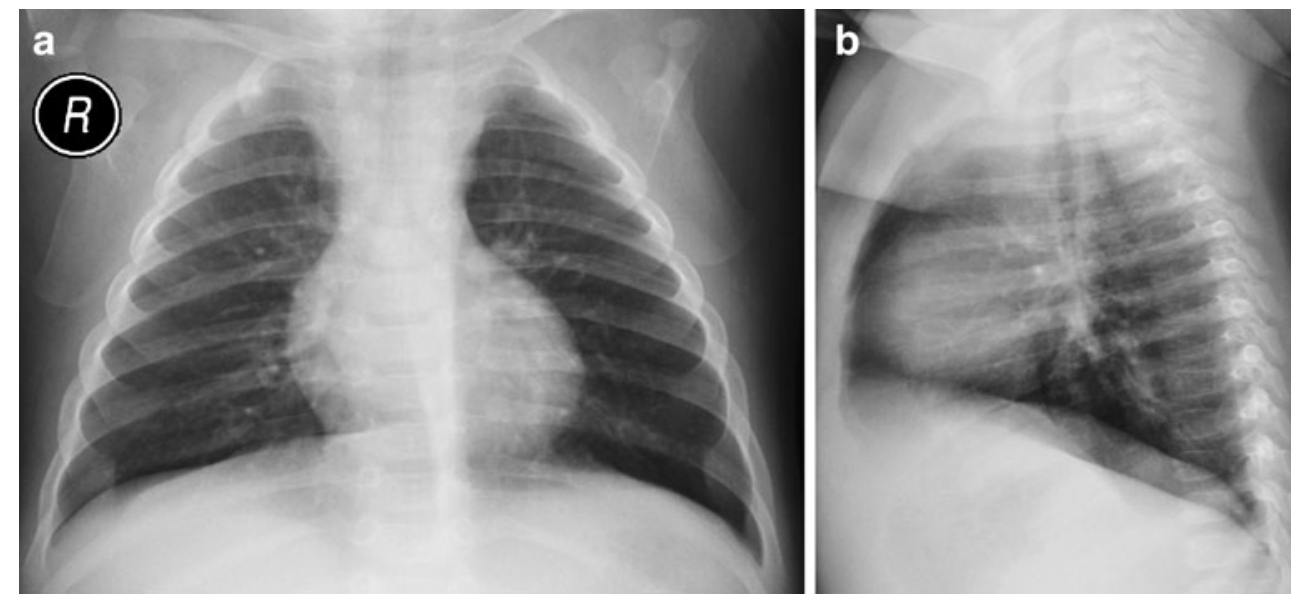


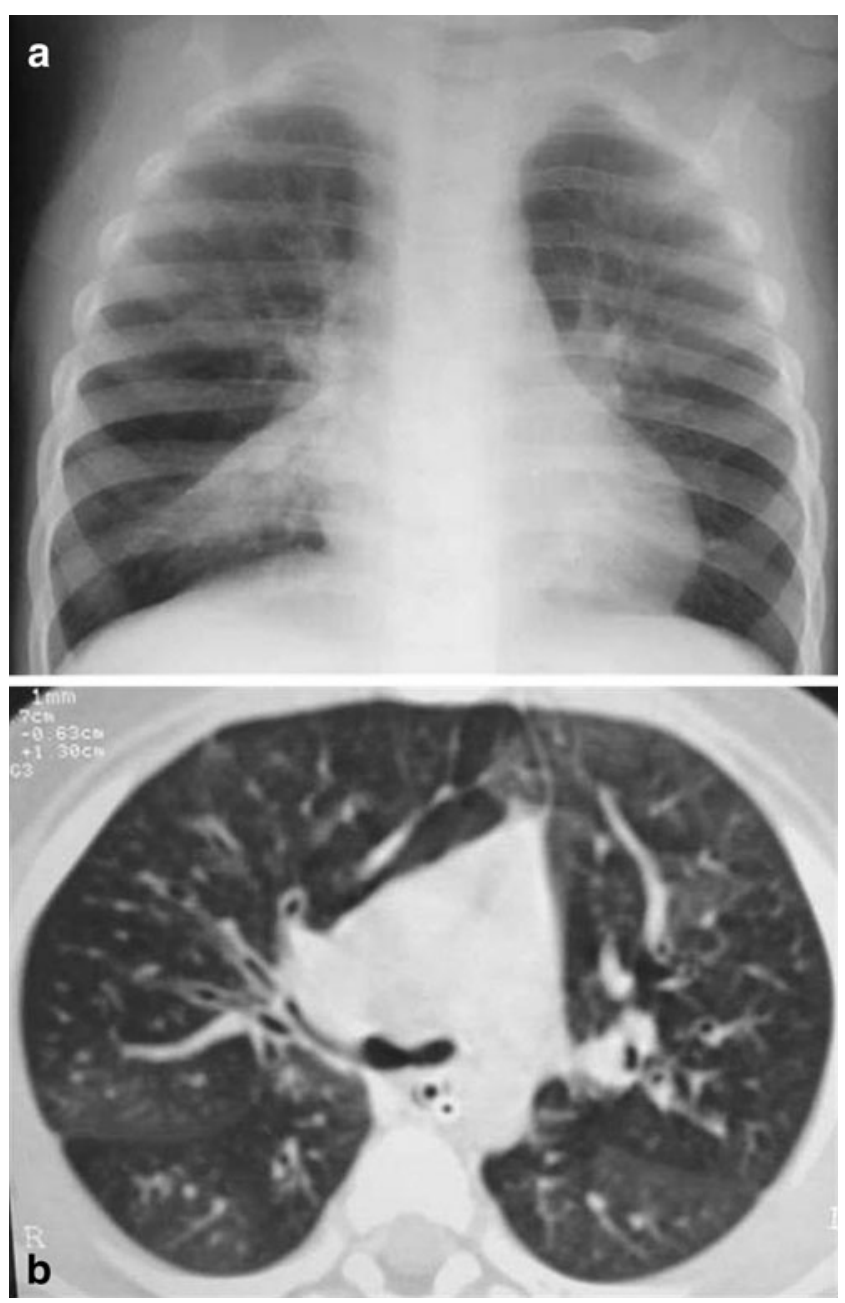

Fig. 2 Adenovirus bronchiolitis in an 11-month-old boy. a Chest radiograph demonstrates bilateral pulmonary hyperaeration and right middle lobe atelectasis. b Axial CT shows mosaic attenuation pattern, diffuse cylindrical bronchiectasis and right middle lobe atelectasis

should not be confused with postinfectious bronchiolitis obliterans. It shares many of the clinical features of pneumonia but is not of infectious etiology [10,11].

\section{Bacterial pneumonia}

Bacterial pneumonia is generally characterized by consolidation and filling of alveolar air spaces with exudate, inflammatory cells and fibrin. The first phase is characterized by active hyperemia and engorgement of the arterial blood vessels. Edematous fluid, which might be seen in the alveoli, contains few exudative cells. In the next stage, neutrophils and fibrin material fill the alveoli, and massive extravasation occurs through interalveolar pores (pores of Kohn), producing a homogeneous opacity. Fibrin and exudative cells accumulate, appearing on radiographs as a clear zone adjoining the alveolar and acinar cells $[9,12,13]$. If the process extends to the pleural space, associated pleural effusion is present.

Bronchopneumonia is a form of bacterial pneumonia that begins as a peribronchiolar inflammation involving many lobes and spreads to the adjacent parenchyma, causing patchy nodules and consolidation. Streptococcus pneumoniae is a common infectious agent but pathogens like Haemophilus influenzae, Staphylococcus aureus and gram-negative enteric bacteria can cause similar clinical syndromes. The typical chest radiograph reveals homogeneous or fluffy lobar consolidation, but diffuse bilateral inhomogeneous opacities extending peripherally also can be seen, both with and without associated pleural fluid (Fig. 6). As opposed to the other bacterial pneumonias,
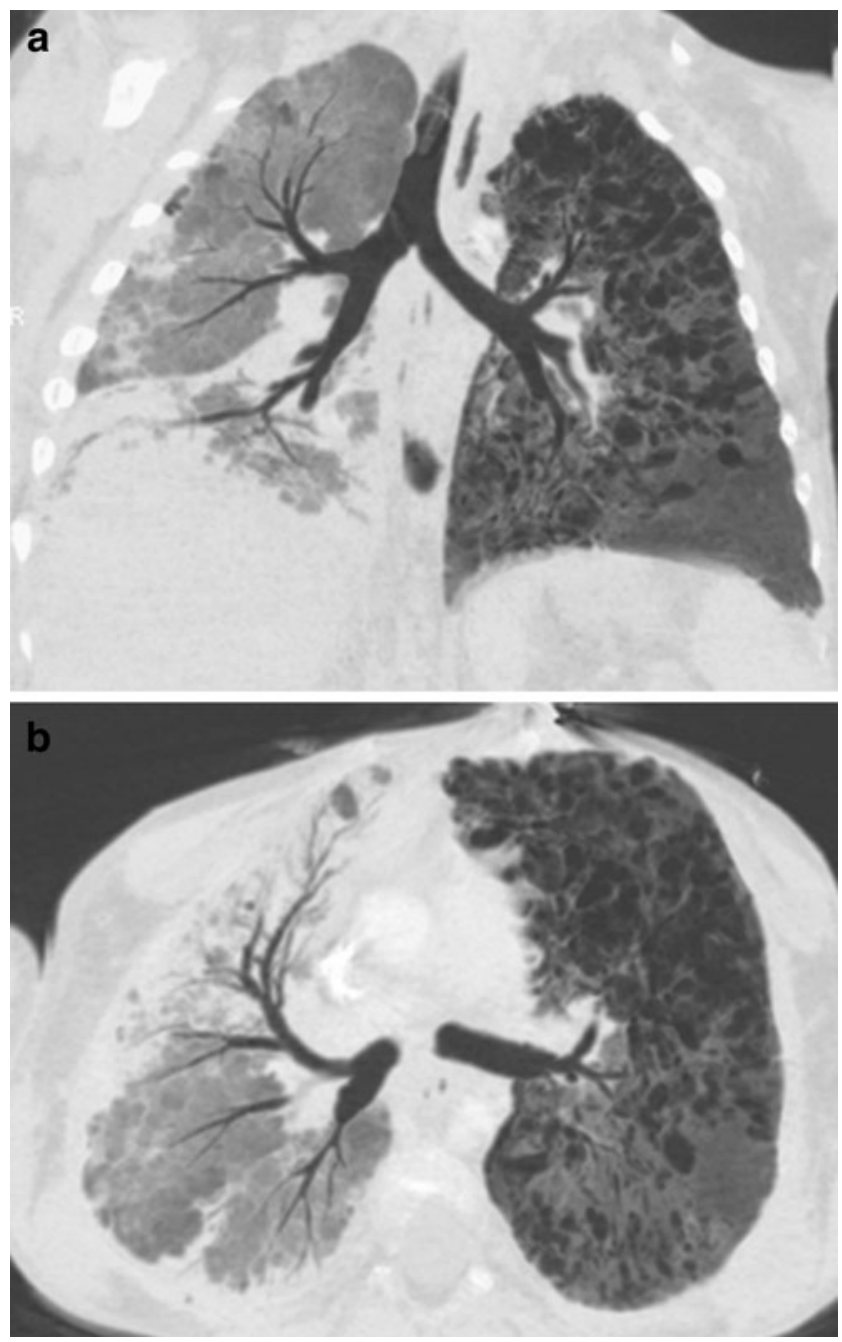

Fig. 3 H1N1 infection in a 7-year-old boy. Coronal MPR (a) and axial CT (b) show volume reduction, ground-glass opacities and parenchymal consolidations in the right lung. Left lung shows a mixed pattern of cystic-like hyperinsufflation lesions and ground-glass opacities 

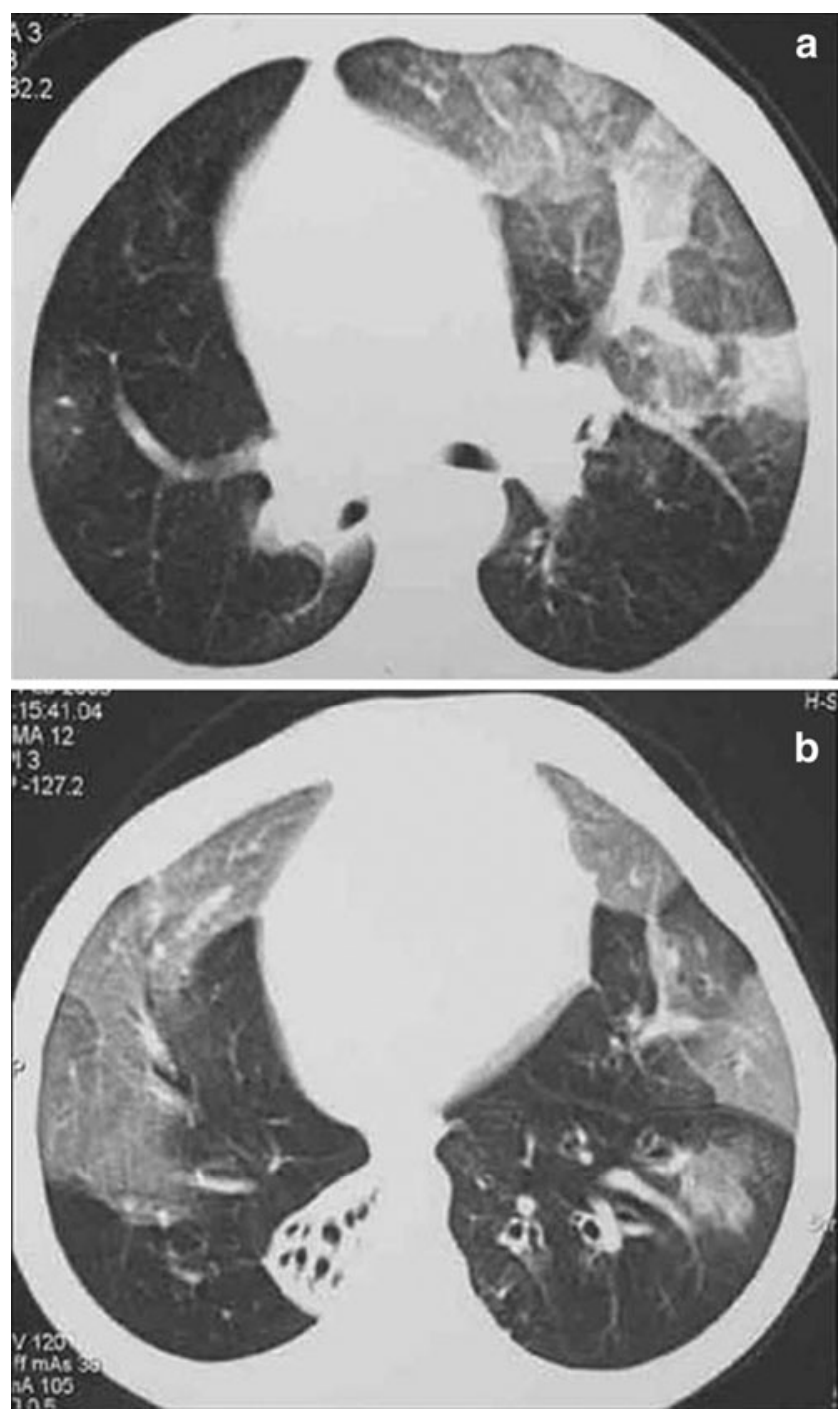

Fig. 4 Postinfectious bronchiolitis obliterans after adenovirus infection. a, b CT shows extensive bilateral mosaic perfusion pattern with areas of decreased attenuation and vascularity, peribronchial thickening and complete right lower lobe atelectasis

Streptococcus pneumoniae more commonly causes lobar and segmental pneumonia.

Round pneumonia has an atypical radiographic appearance of pulmonary bacterial infections. It is caused most often by $S$. pneumoniae. It is rare in adults and occurs in less than $1 \%$ of children (usually younger than 8 years of age) but is seen more frequently in the young because of the underdeveloped pores of Kohn and the absence of canals of Lambert characteristic of this age group. The radiographic finding usually occurs early in the disease course, and for this reason cough and respiratory symptoms can be absent in the initial presentation. The usual presentation is an area of focal nodular consolidation, often solitary, with well-defined borders and mass effect. It frequently occurs in the lower lobes, posteriorly $(>80 \%)$ and inferiorly (>70\%) [14] (Fig. 7).

Pneumonias have been classified as atypical based largely on the observation of clinical (low white count, little or no fever, etc.) and imaging findings disproportionate to the clinical condition. Legionella species, Mycoplasma pneumoniae and Chlamydia pneumoniae are the most common etiologies of atypical pneumonias. Reticulonodular infiltrate in one lobe is a usual presentation of mycoplasmal pneumonia. Other radiographic features are lobar consolidation or pseudoconsolidation (Fig. 8), atelectasis, bilateral parahilar peribronchial opacities that resemble butterfly wings, bilobar reticular pattern, pleural and pericardial effusion and hilar lymphadenopathy $[12,13]$. Many of these features can be seen in viral pneumonia as well. To distinguish these pneumonias one needs to recognize the clinical features that don't appear to match the radiograph, such as the degree of respiratory distress or the unusual laboratory findings. For example, Chlamydia trachomatis (or chlamydophila) affects young infants in the first 4 months, and frequently a history of conjunctivitis and a staccato cough are observed. The history will reveal a vaginal delivery and a mother with vulvovaginitis. Chest radiograph shows bilateral reticular interstitial involvement, hyperinflation and bronchial wall thickening.

\section{Complicated bacterial pneumonia}

The three most common complications of bacterial pneumonia are pulmonary necrosis, pulmonary abscess and empyema. Children who have complicated pneumonias might require an imaging evaluation by US or chest CT $[15,16]$. Necrotizing lobar lesions result from thrombotic occlusion of alveolar capillaries associated with adjacent inflammation, resulting in ischemia and eventually necrosis. The typical CT findings of necrotizing pneumonia are cystic heterogeneous areas of necrosis within solid consolidation and non-enhancing lung parenchyma (Fig. 9) [17].

Pulmonary abscess is a necrotic parenchymal cavity filled with pus. CT imaging shows a cystic area within consolidated lung parenchyma but with contrast rim enhancement (Fig. 10). Chest CT with intravenous contrast administration can be very useful in atypical, complicated pneumonia presentations, although it should not be performed routinely. CT is better than chest radiograph for detecting lobar lesions such cavitary necrosis, early abscess formation, chest tube placement, fluid loculation, empyema, bronchopleural fistulas and pericardial effusions. CT also helps define lesions that might require early surgical intervention $[15,16]$. Chest sonography, however, is mainly used to demonstrate the internal echogenicity of pleural 

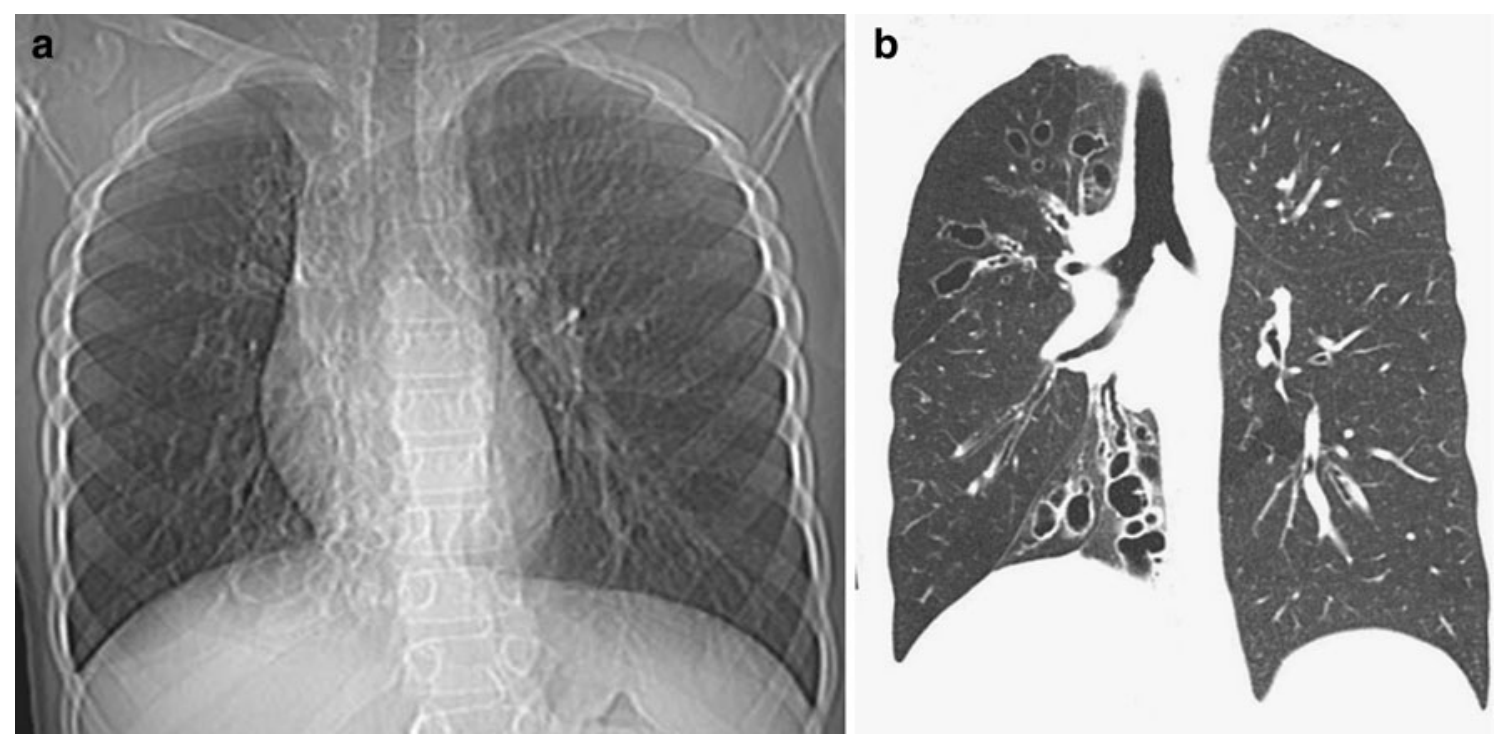

Fig. 5 Swyer-James-MacLeod syndrome in an 11-year-old boy. CCR (a) and coronal MPR CT (b) show hyperlucency and decreased vascularity of the right lung. The mediastinum is shifted to the right,

effusions and the presence of septations, although it can be useful for detecting parenchymal lesions.

\section{Complications after pneumonia}

Pneumatoceles and bronchiectasis are the most common postinfectious sequelae of bacterial pneumonia. Pneumatoceles are thin-walled cystic lucencies with or without septations that develop within the lung parenchyma, occurring after an acute pneumonia most often caused by $S$. aureus (Fig. 11). consistent with decreased right lung volume. Bronchiectasis is more evident in the upper and lower right lobes

Bronchiectasis is an abnormal irreversible dilation of the proximal and medium-size bronchi caused by weakening or destruction of the muscular and elastic components of the bronchial walls. The more commonly acquired forms occur in older children after destructive pulmonary infections that cause impaired clearance of bronchial secretions, or in cases of superimposed bacterial infections in children with previous conditions such as cystic fibrosis. Bronchiectasis can be observed as thickening of the bronchial walls and marked dilatation of the bronchial channel on both radiographs and chest CT.
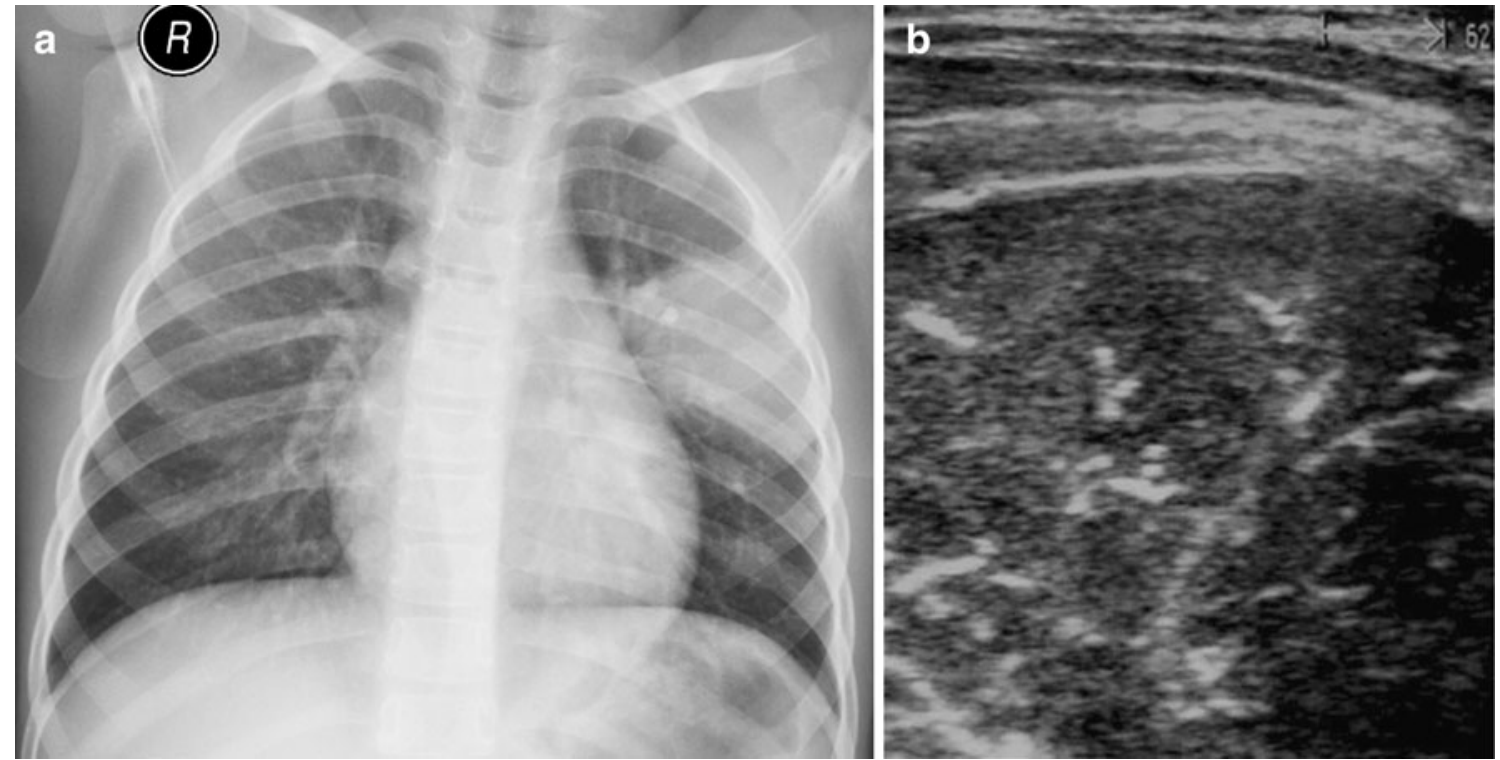

Fig. 6 Lobar pneumonia. a Chest radiograph frontal view demonstrates focal area of consolidation in the left upper lobe. b US shows the typical hepatization appearance of lung parenchyma with air bronchograms 
Fig. 7 Round pneumonia in a 9-year-old boy. Frontal (a) and lateral (b) chest radiographs show a well-defined mass in the apical segment of the left lower lobe on the lateral view. Chest radiograph obtained 2 weeks later revealed complete resolution
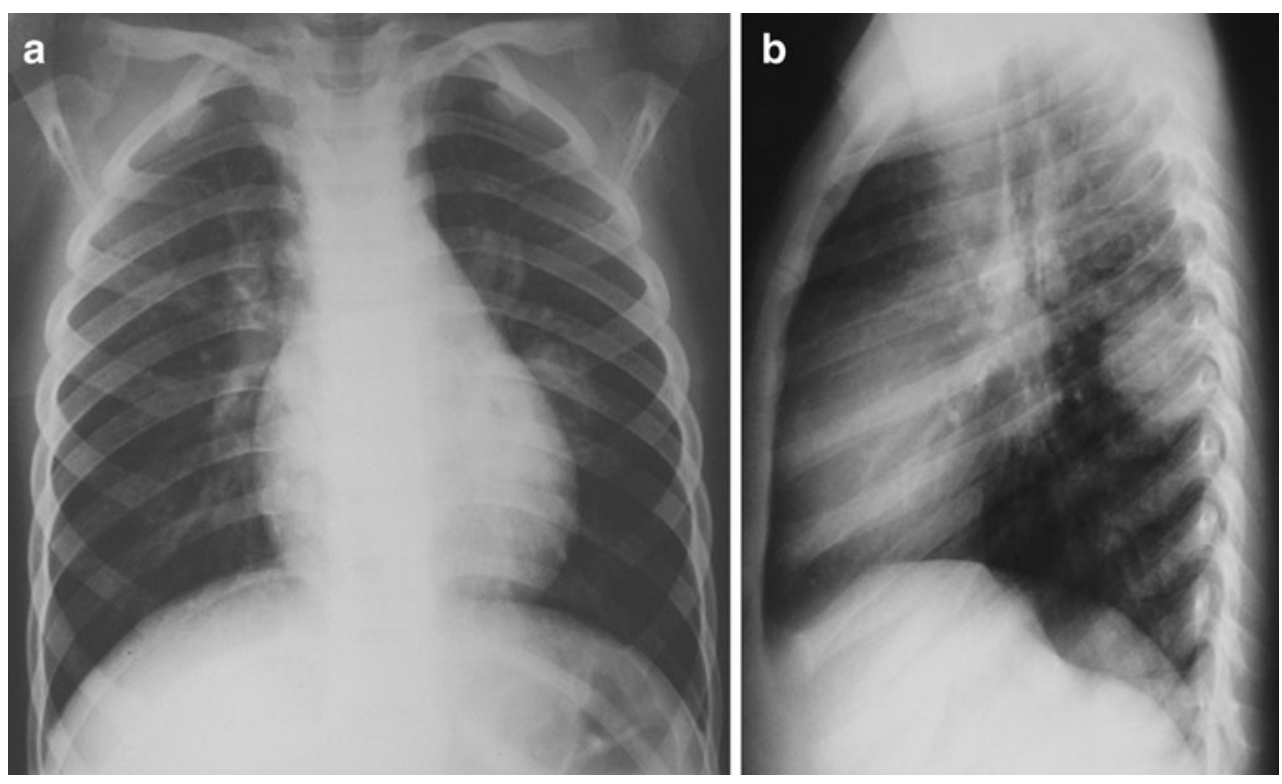

\section{Tuberculosis}

Mycobacterium tuberculosis causes tuberculosis, and its incidence is increasing worldwide. Sixty percent of new childhood infections occur in children younger than 5 years old [2]. Pulmonary tuberculosis in infancy and childhood is classically related to an inhaled innoculum. There is a small, peripheral primary focus that leads to lymphadenopathy asymmetrically distributed in paratracheal, hilar and/or subcarinal areas. The right side is more commonly affected than the left because of the usual pattern of lymphatic circulation within the lungs [1, 18-20]. Enlarged lymph nodes with lobulated, sharp or ill-defined borders can be partially or completely calcified. CT is reserved for complicated cases, not those with a positive skin test.
Lymph nodes on CT are dense soft-tissue masses with homogeneous or ring-like enhancement and lowattenuation centers. Inflamed nodes sometimes erode the bronchial wall and discharge caseous material into the bronchus (Fig. 12). This results in bronchogenic spread, characterized as centrilobular or branching centrilobular opacities. Coalescence of the centrilobular opacities results in focal areas of bronchopneumonia [1,21]. Sporadically, the primary parenchymal lesion continues to enlarge, resulting in focal pneumonitis or lobar pneumonia with thickening of the overlying pleura without distinct hilar lymphadenopathy. It is helpful to divide the disease into subtypes. Primary tuberculosis, which sometimes progresses to a symptomatic infection, is often indistinguishable from other forms of bronchial pneumonia. Enlarged
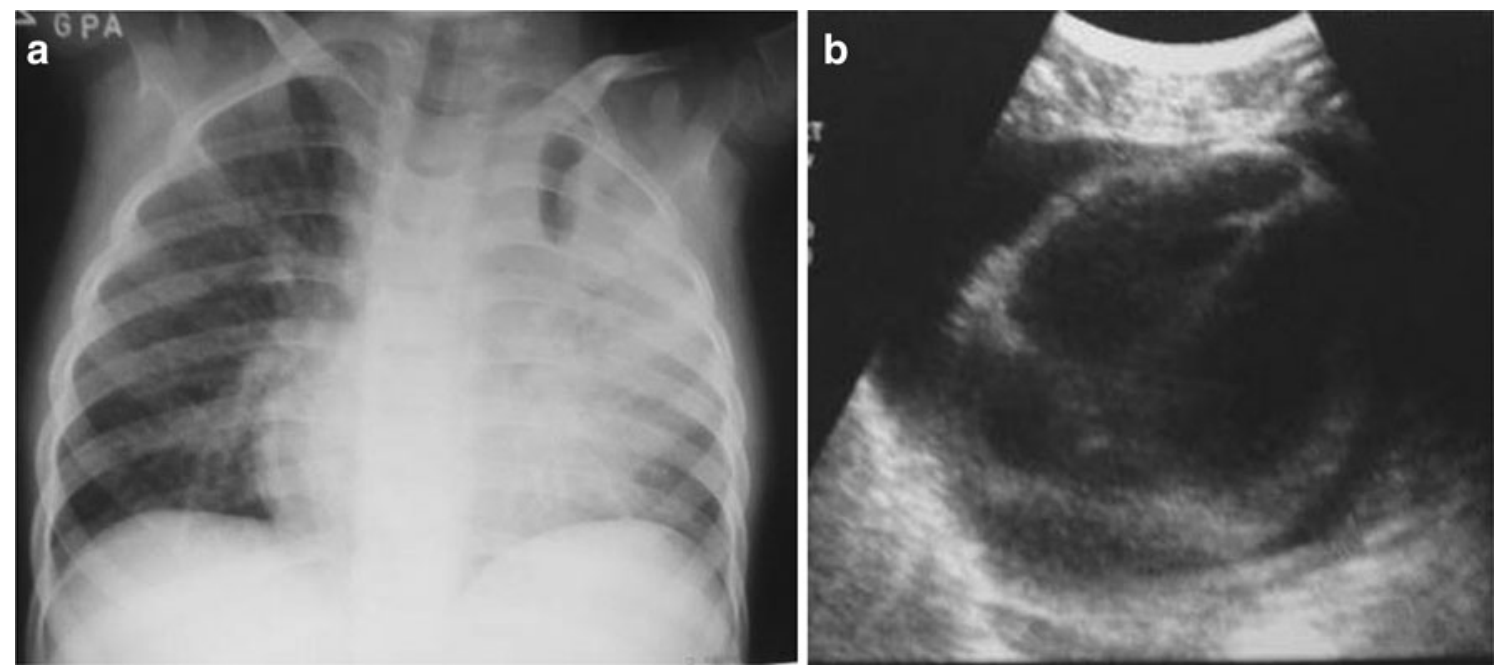

Fig. 8 Mycoplasma pneumonia in a 3-year-old girl with low fever and cough. a Frontal chest radiograph shows left upper lobe consolidation. b Pericardial effusion is seen on US image 


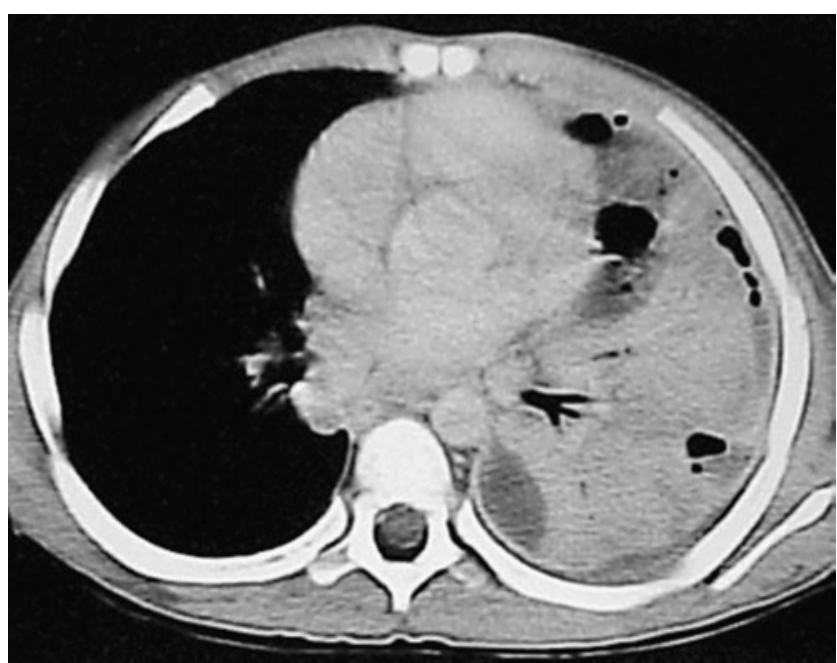

Fig. 9 Necrotizing pneumonia. Axial CT after contrast administration shows extensive left lower lobe consolidation with a decreased enhancement area suggesting necrosis. Note the small air-filled cavities

lymph nodes are a hallmark but are not specific. Lymph node involvement is a dominant feature in all subtypes and should raise suspicion for this infection.

In cases of tuberculosis, progressively destructive liquefaction of lung parenchyma leads to formation of a primary cavity or more extensive pulmonary necrosis (Fig. 13). Hematogenous spread can also develop with a miliary pattern of small, well-defined nodules of up to several millimeters in diameter uniformly distributed through the lungs $[1,22]$.

It is important as well to recognize healed TB as latent infection can reactivate. The immune response to the initial infection leads to calcification of the healing lung as well as

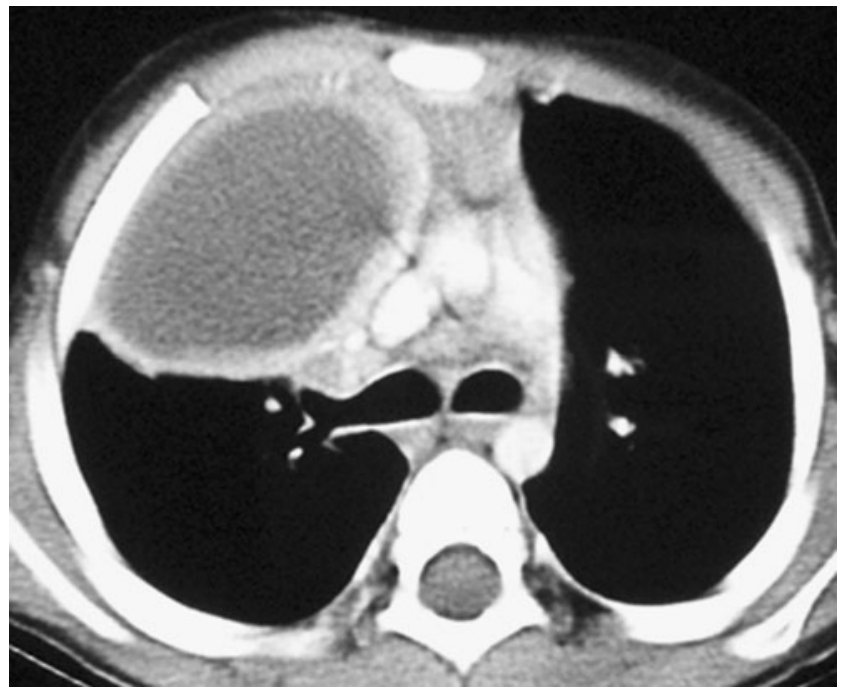

Fig. 10 Pulmonary abscess. Contrast-enhanced axial CT of the chest shows a large cavitary lesion in the right upper lobe with a relatively thick wall. The cavity has a smooth inner margin
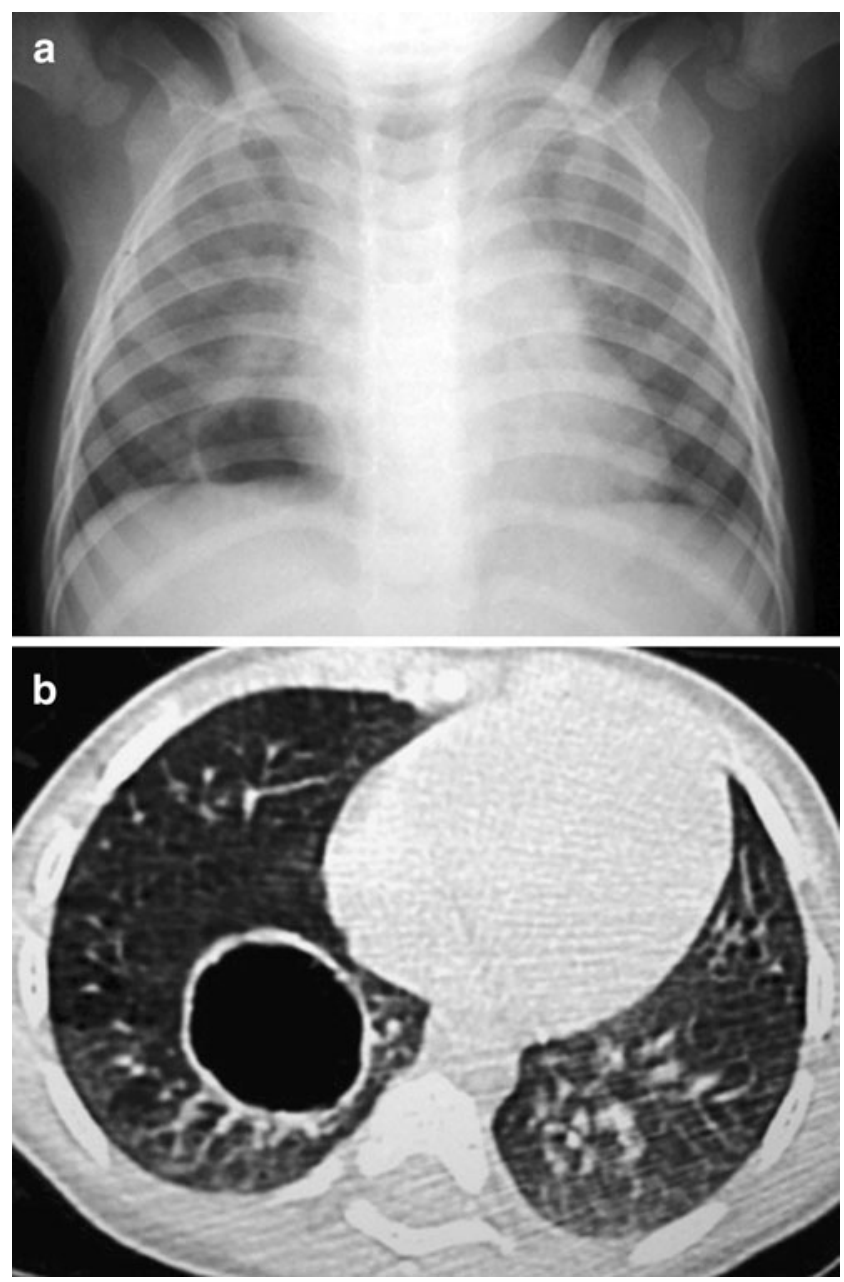

Fig. 11 Pneumatocele in an infant. Conventional chest radiograph (a) and axial CT (b) show a thin-walled cavity in the right lower lobe

infected lymph nodes. This observation is important, as the child's immune function can change. In children who have newly diagnosed HIV infection or are undergoing chemotherapy for malignancy or transplantation, it might be important to treat latent TB before it reactivates [22-24].

\section{Fungal pneumonia}

Histoplasmosis and aspergillosis are the most common fungal pneumonias in children, most often in the immunesuppressed group (see below) $[25,26]$. Histoplasmosis is a systemic mycosis caused by a small fungus, Histoplasma capsulatum, whose natural habitat is soil contaminated by bat or bird excrement. It is endemic in some geographical regions, such as the Ohio River Valley. Human infection occurs when airborne spores of $H$. capsulatum are inhaled. The most common imaging features are diffuse reticulonodular opacities with small and diffuse pulmonary nodules (Fig. 14). Usually it affects both lungs as well as hilar and 

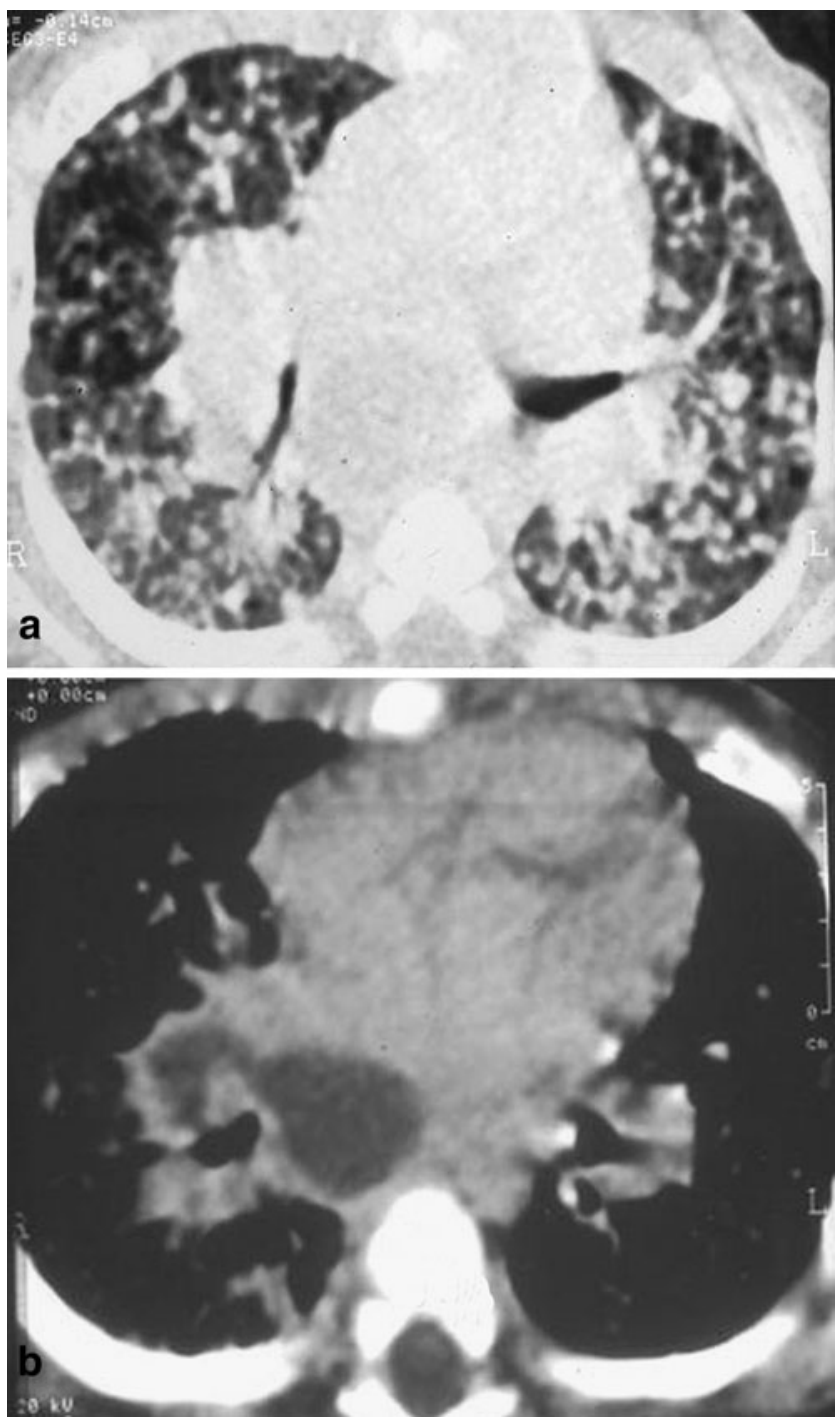

Fig. 12 Pulmonary tuberculosis in a 6-month-old boy. Chest CT, lung (a) and mediastinal (b) windows show right hilar lymph node with hypodense center (caseum) and fistulous tract to the main bronchus causing bronchogenic spread, with multiple pulmonary nodules of different sizes in both lungs

mediastinal lymph nodes. This miliary pattern can be identical to miliary tuberculosis and blastomycosis [25]. Pulmonary nodules heal, leaving residual granulomata that undergo central calcification. Mediastinal lymph nodes can also exhibit central caseation, peripheral enhancement and, frequently, calcification as in tuberculosis. Another uncommon but severe complication of histoplasmosis is mediastinal fibrosis, which not only causes mass effect in the mediastinum, it can compress major blood vessels or occlude bronchi (Fig. 15) [26, 27].

Aspergillosis refers to any infection caused by several species of Aspergillus: A. fumigatus, A. flavus, A. niger and $A$. terreus. The fungus is ubiquitous, and asymptomatic colonization is common. Three factors are important to the development of active infection: the virulence of the fungus, the type and amount of exposure, and the immune status of the child. The spectrum of lung diseases ranges from allergic reactions, colonization of a preexisting lung cavity, or invasion and destruction of lung tissue, to progressive vascular invasion with involvement of the brain, skin and other organs [28-30].

Allergic bronchopulmonary aspergillosis (ABPA) affects children with asthma or cystic fibrosis. The disease is a hypersensitivity reaction to the fungus; the findings are secondary to the reaction to the allergen. This form of the infection occurs most frequently in children with cystic fibrosis. It is important to recognize that prompt therapy can prevent progression of the child's bronchiectasis. ABPA
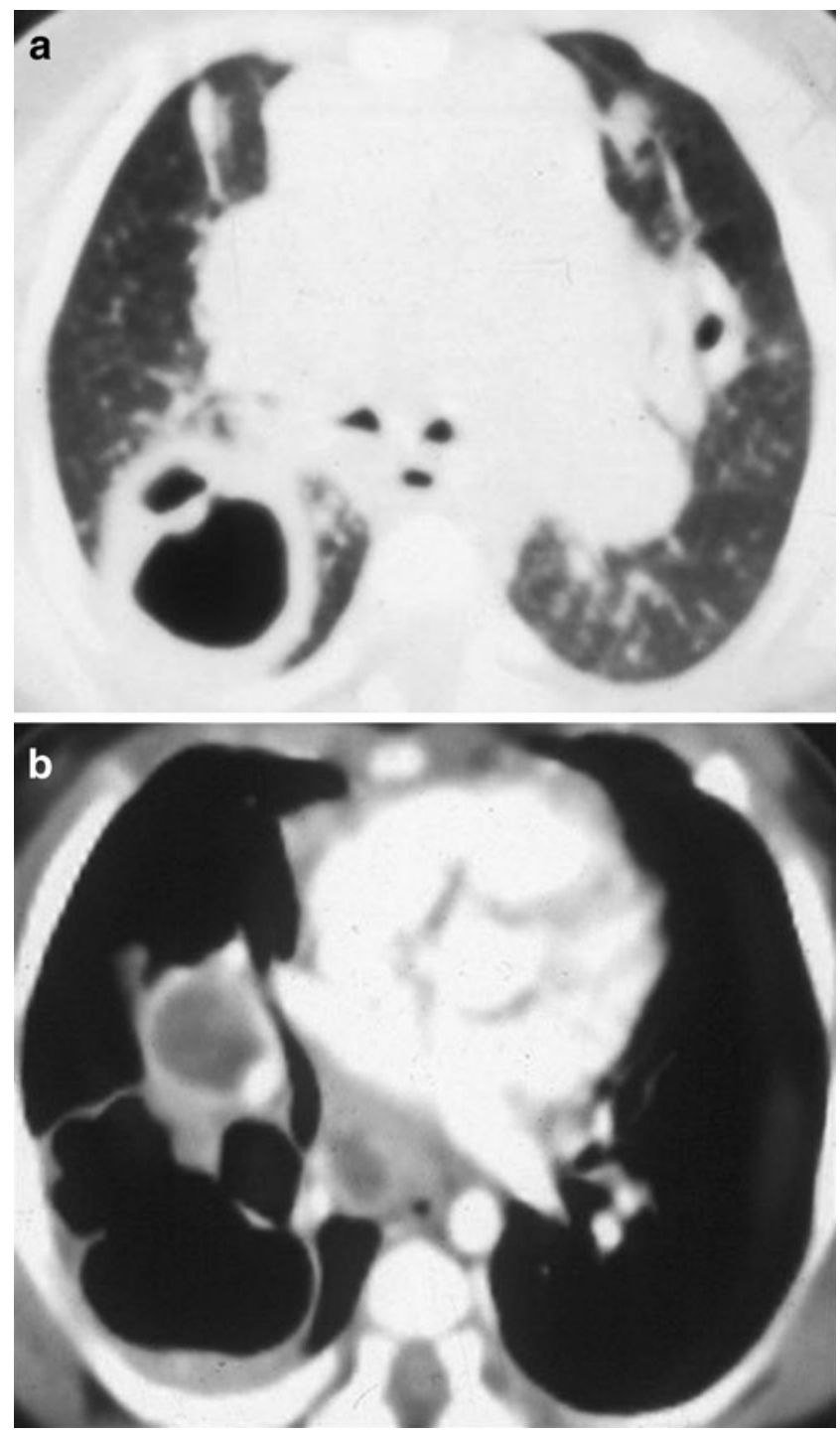

Fig. 13 Cavitary pulmonary tuberculosis in a 2-month-old boy. Lung (a) and mediastinal (b) axial CT views show a large mediastinal and hilar lymphadenopathy with a cavity lesion in the right lung. Enhanced scan (b) shows cavitary lesion and rim enhancement tuberculosis lymph nodes 

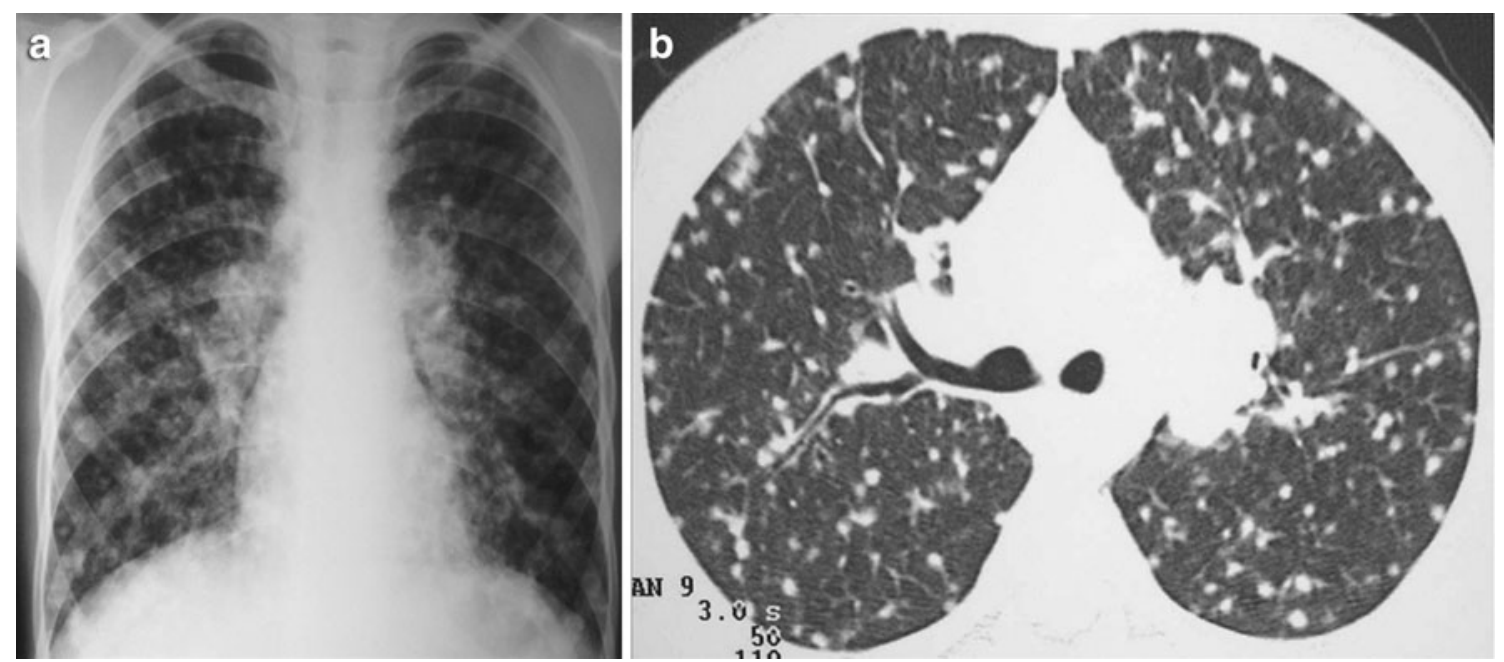

Fig. 14 Histoplasmosis in a 9-year-old boy. Chest radiograph (a) and CT (b) show bilateral reticulonodular opacities with multiple diffuse small nodules as well as hilar lymphadenopathies

should be considered in $\mathrm{CF}$ patients with new asthma symptoms and when any new chest radiograph airspace changes are found and when IgE titers increase [30]. The most common CT findings are proximal and segmental bronchiectasis with or without mucous plugs and fleeting consolidations.

Aspergilloma, in which the fungus colonizes a preexisting lung cavity, occurs in individuals with chronic lung disease and pulmonary sequelae such as tuberculosis, bronchiectasis and pulmonary fibrosis. Invasion to lung parenchyma, pleura and fibrosis is minimal, and a fungus ball might be present. Neither fungal vascular invasion nor spread to other organs is observed. The disease can persist for months or years. Chest CT usually reveals a consolidation with an interposed cavitation and an ovoid intracavitary mass (fungus ball, or mycetoma) consisting of fungal hyphae, mucus and cellular debris [28-30]. This infection is often associated with underlying pathologies and other infections. It is not uncommonly found in cavities from TB infection or in congenital bronchopulmonary malformations, such as sequestrations.

Angioinvasive aspergillosis is one of the most common forms of fungal infection among immunocompromised and neutropenic children. The lesions seen on conventional chest radiograph include single or multiple nodules, cavities, and subsegmental or segmental consol-
Fig. 15 Fibrosing mediastinitis in a 17-year-old child. CT scan shows a mediastinal mass surrounding and narrowing the right pulmonary artery
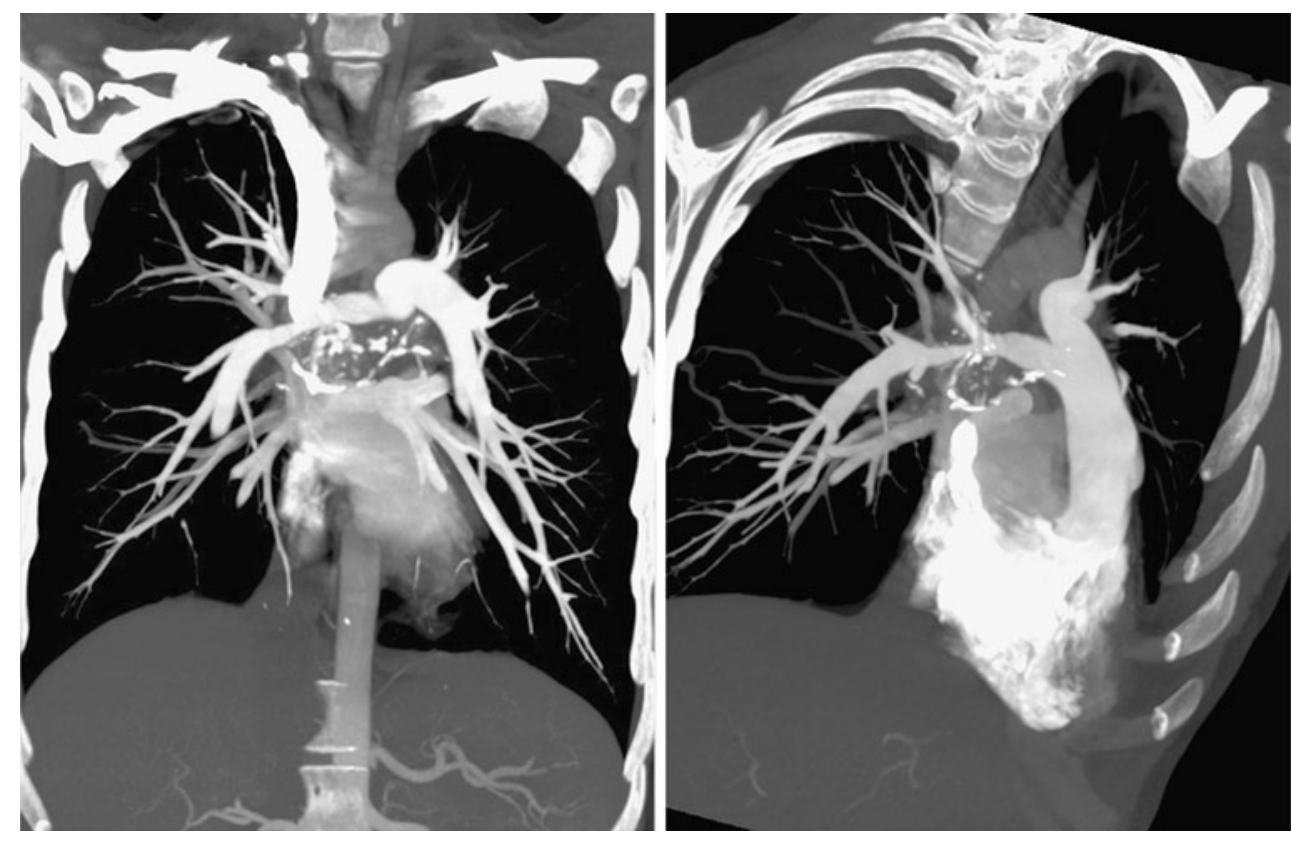
idation. In the initial phase, the most characteristic image seen on CT is the halo sign, an area of low attenuation around the centrilobular nodules that represents edema or hemorrhage (Fig. 16). In later stages, CT might show areas of necrotic tissue and pulmonary sequestration within the surrounding parenchyma, resulting in the air crescent sign [31].

\section{Parasites}

Ascaris lumbricoides can lead to an acute inflammatory lung process caused by the host immunological response to parasite migration. This is called Loeffler syndrome, or acute allergic eosinophilic pneumonia. Chest radiograph and $\mathrm{CT}$ often show transient nonsegmental airspace opacification, which can be unilateral or bilateral, usually with predominantly peripheral distribution. The features are not specific for this etiology of allergic eosinophilic pulmonary infiltration.

Echinococcosis is a parasitic disease usually caused by Echinococcus granulosus, which affects humans through accidental ingestion of parasite eggs released in the feces of definitive, carnivorous hosts, such as sheep, dogs and cats. Especially in children, infection can also occur through direct contact with infected domestic animals. Echinococcus eggs hatch into larvae in the duodenum, then migrate through the portal system to the liver and to pulmonary alveolar lung capillaries, where they develop into spherical or oval cysts. The liver is the primary site of infection in $75 \%$ of patients. The CT scan might show single or multiple cysts, which can present as daughter cysts or floating cysts with internal membranes (water lily sign) (Fig. 17) [32].

In addition amebiasis, malaria and protozoa infections along with toxoplasmosis are well recognized to cause

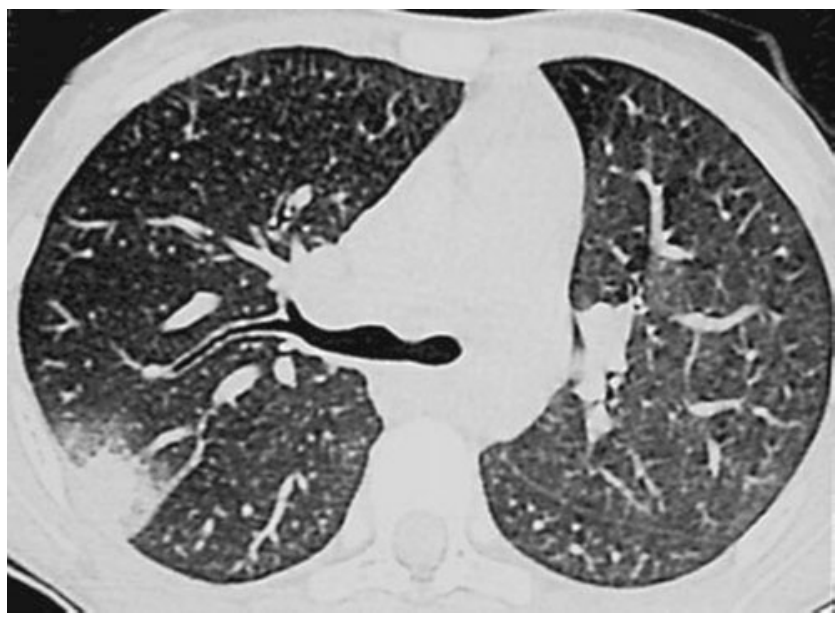

Fig. 16 Axial CT shows the typical halo sign in an immunocompromised child with invasive aspergillosis pulmonary disease. The increase in HIV/AIDS as a cause of immunosuppression has increased the incidence of parasitic infection and pulmonary complications worldwide. The radiographic diagnosis is not specific for any of these diseases and their recognition relies upon an astute clinician and serological evaluation with polymerase chain reaction (PCR) testing.

\section{Pulmonary infection in immunocompromised children}

Pulmonary infections are one of the most significant causes of morbidity and mortality in immunocompromised children. Regardless of the immunodeficiency cause, the most common complications are infections caused by bacterial, viral or opportunistic pathogens [5, 33-35]. Immunodeficiency can be divided into major groups of primary and secondary. A simple model of the immune system is that it is mediated by two major mechanisms: humoral and cellular. Defective development of B cells results in humoral immunity abnormalities, while defective development of $\mathrm{T}$ cells causes cellular immunity problems. Children with impairment of cellular or humoral immune mechanisms or both experience a higher propensity for specific infections. Humoral immune impairments usually result in bacterial infections, while cellmediated immune impairments make children more prone to opportunistic, viral and mycobacterial infections [34]. This applies to children with primary immunodeficiency syndromes and secondary immunodeficiency diseases, including AIDS. Most primary immunodeficiencies are humoral deficiencies, with IgA deficiency, X-linked agammaglobulinemia and common-variable immunodeficiency the best known examples. These children are prone to repeated bacterial infections with associated pneumonia leading to postinfectious bronchiectasis (Fig. 18). A much smaller group of primary immunodeficiencies is caused by changes in cellular immunity (DiGeorge syndrome and severe combined immunodeficiency) or in phagocyte activity like chronic granulomatous disease. DiGeorge syndrome, which is also called thymic aplasia or hypoplasia, is a typical example of a primary $\mathrm{T}$ cell deficiency with abnormal development of the thymus, parathyroid glands and heart. Children with this syndrome are susceptible to opportunistic organisms and to graft-versus-host disease from blood transfusions [34].

Chronic granulomatous disease is a genetic syndrome characterized by an abnormality in an oxidative metabolic response during phagocytosis and resulting in bacterial and fungal infections. Children with chronic granulomatous disease develop recurrent infections resulting in granuloma formation. These granulomatous lesions are seen in the skin, lungs, liver and lymph nodes. The onset of symptoms usually occurs early in life. Chest radiograph and CT findings show 
Fig. 17 Pulmonary echynococcal cysts. Frontal (a) and lateral (b) chest radiographs show welldefined round masses in the right lower lobe. Chest US (not shown) demonstrated the cystic nature of the lesions
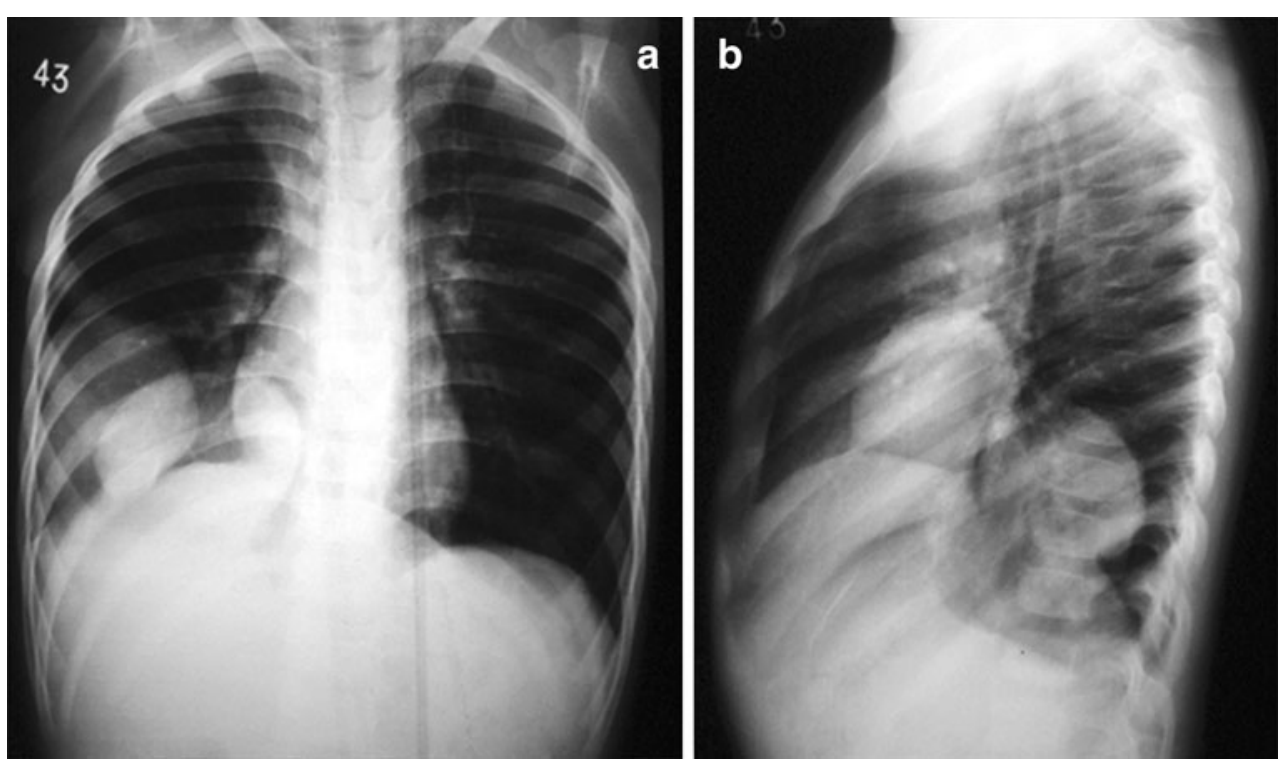

chronic or recurrent pneumonia, usually by Aspergillus or Candida organisms. Hilar and mediastinal adenopathy, pleural reaction and chest wall invasion can be also present $[5,36]$.

Hyper-IgE syndrome (HIES, Job syndrome) is a complex primary immunodeficiency disorder characterized by a spectrum of abnormalities related to the immune system, bones, connective tissue and teeth. HIES was initially defined as a triad of clinical problems involving skin boils, severe episodes of pneumonia and very high serum IgE levels. Recurrent staphylococcal cutaneous lesions and

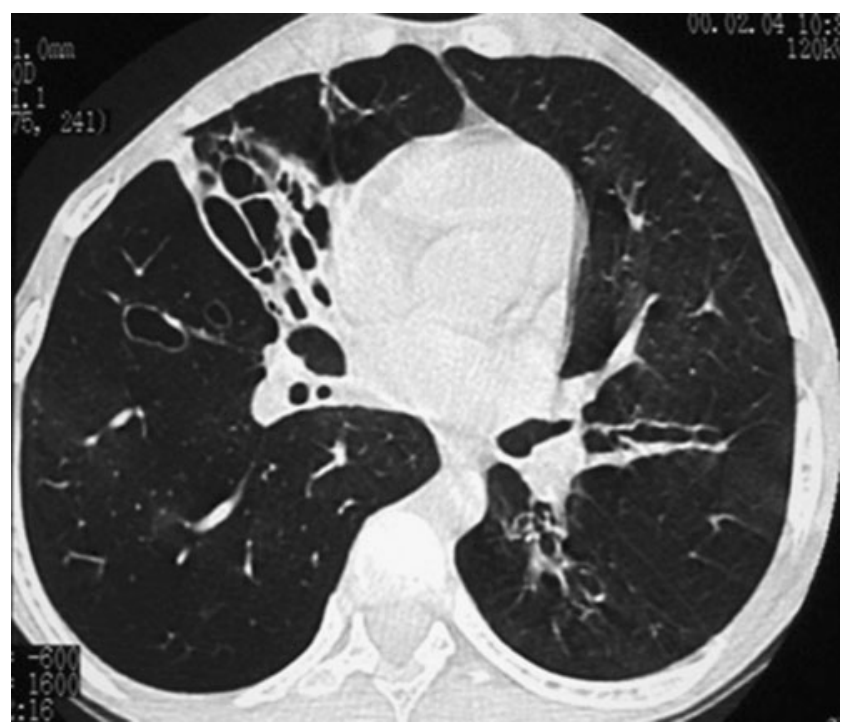

Fig. 18 Immunodeficiency. Axial CT shows diffuse bilateral hypoattenuation with decreased vascularity and postinfectious bronchiectasis in a child with IgA deficiency after repeated infections and associated right upper lobe atelectasis pneumonia beginning in infancy and resulting in lung cyst formation with variable persistence and expansion are the typical findings [37-39] (Fig. 19).

Secondary immunodeficiency affects a mixed group of neutropenic children with previous chemotherapy or radiotherapy, immunosuppressed hosts after an organ or bone marrow transplant, and children with HIV/AIDS [2, $5,40,41]$. In the second group, the deficiency occurs to a greater extent than in cellular immunity. However, humoral immunity can also be compromised in neutropenic and severely malnourished children. Children who are predominately neutropenic as a result of chemotherapy or who are in the early phase after a bone marrow transplant with or without incipient graft-versus-host disease are at risk for infection by gram-negative bacteria such as Pseudomonas aeruginosa or by gram-positive bacteria such as $S$. aureus. Fungal pneumonias caused by Aspergillus or Candida species can also occur. Pulmonary nodules are the most frequent finding of candidiasis lung infection on chest CT. These nodules might present the halo sign or excavation. Centrilobular lesions or a mixed pattern can also occur [40, 41].

AIDS is caused by a retrovirus whose main characteristic is the development of immunosuppression and subsequent opportunistic infections and malignancies. Vertical transmission is the usual form of infection in the pediatric population. Lung infection is the leading cause of morbidity and mortality, causing 50 percent of deaths. Viral and bacterial pneumonia are the most frequent infections at diagnosis [3, 42]. The most frequent agents are Streptococcus pneumoniae, Haemophilus influenzae, respiratory syncytial virus and adenovirus. Children are prone to having severe presentations, and bacterial pneumonias are usually associated with cavitations, abscesses and empyema [40]. 

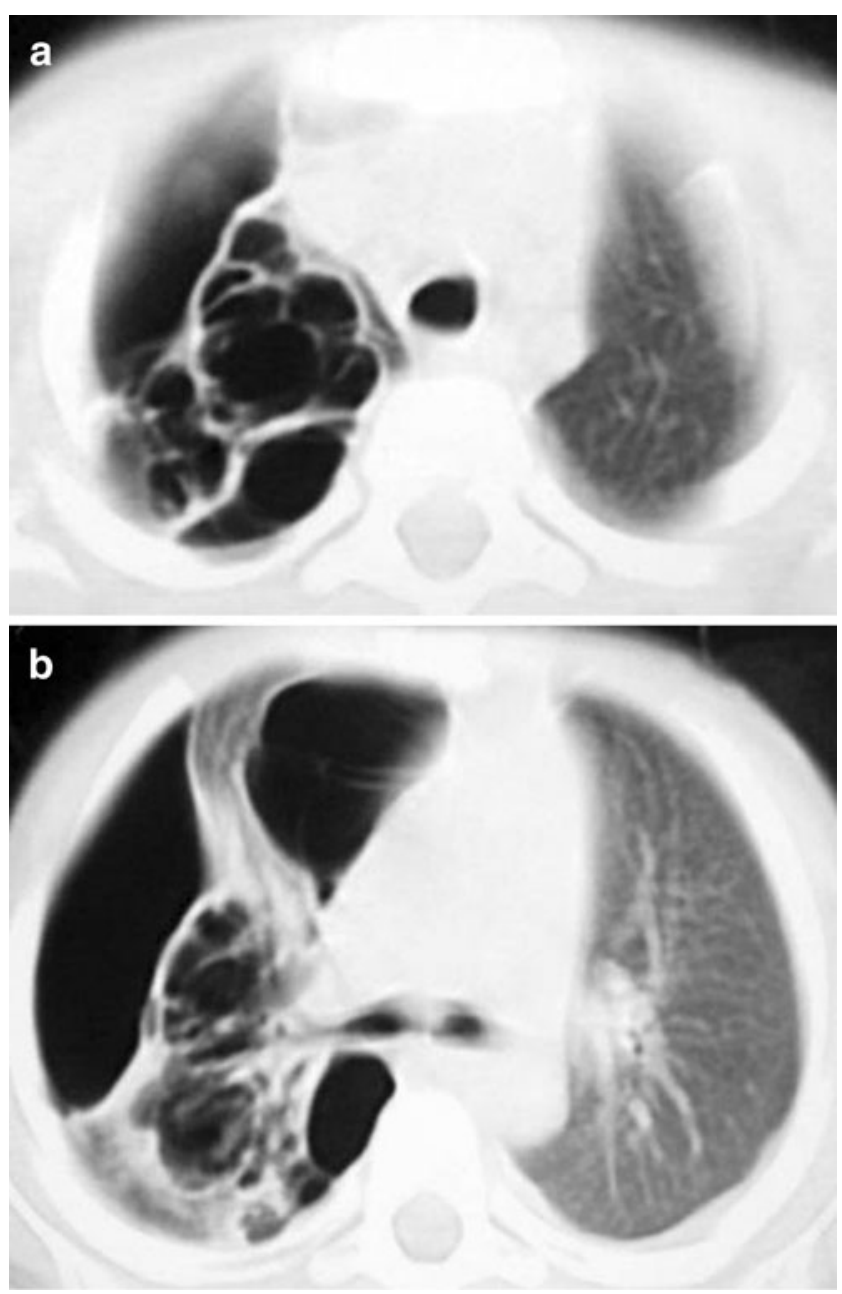

Fig. 19 Hyper-IgE syndrome. Axial chest CT $(\mathbf{a}, \mathbf{b})$ shows persistent multiple large pneumatoceles, expanding cystic lesions and pneumotorax of right lung

Children with AIDS are also susceptible to fungal and mycobacterial infections. Pneumocystis jirovecii is the most common opportunistic infection in infected babies without prophylaxis. P. jirovecii has a high mortality rate during the initial episode. The most common chest radiograph and CT findings are diffuse reticular interstitial opacities that can progress to massive alveolar consolidations resulting in acute respiratory distress syndrome in infants (Fig. 20). Postinfection cavitations and bronchiectasis are typical after recurrent infections. Cryptococcus neoformans infection is rare and has unusual presentations in HIV-infected children. The spectrum of disease ranges from asymptomatic pulmonary lesions to disseminated infection with necrotizing pneumonia, pleural effusion and meningoencephalitis [43] (Fig. 21).

Mycobacterium tuberculosis infection can mimic infections seen in immunocompetent children, but more severe
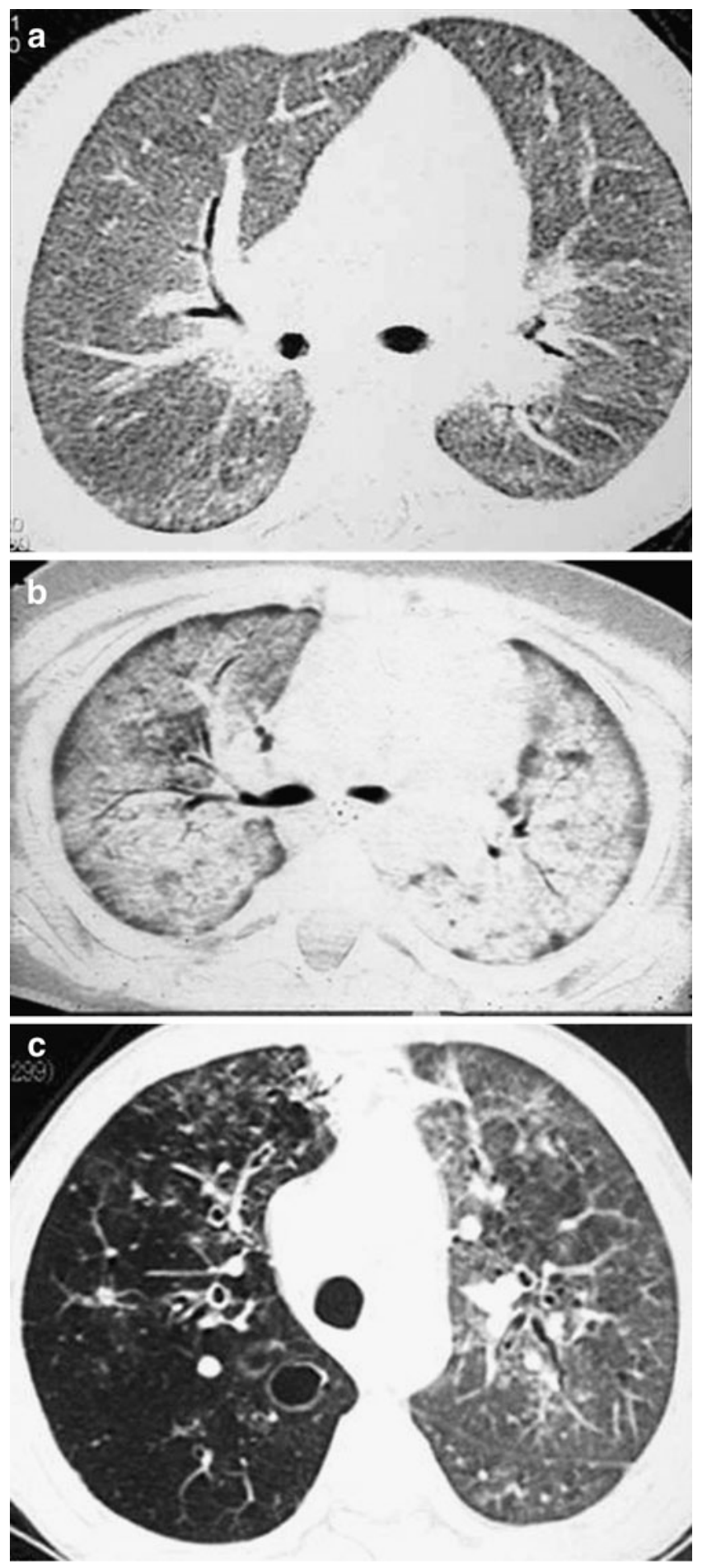

Fig. 20 Pneumocystis jirovecii infection. Axial CT views. a Diffuse bilateral reticular interstitial opacities and (b) ground-glass opacities with progressive consolidations from ARDS in two HAART-naïve HIV-positive infants. c An older child with a right hyperlucent lung with small cavitations and incipient bronchiectasis and a left lung with ground-glass pattern after previous recurrent infections 


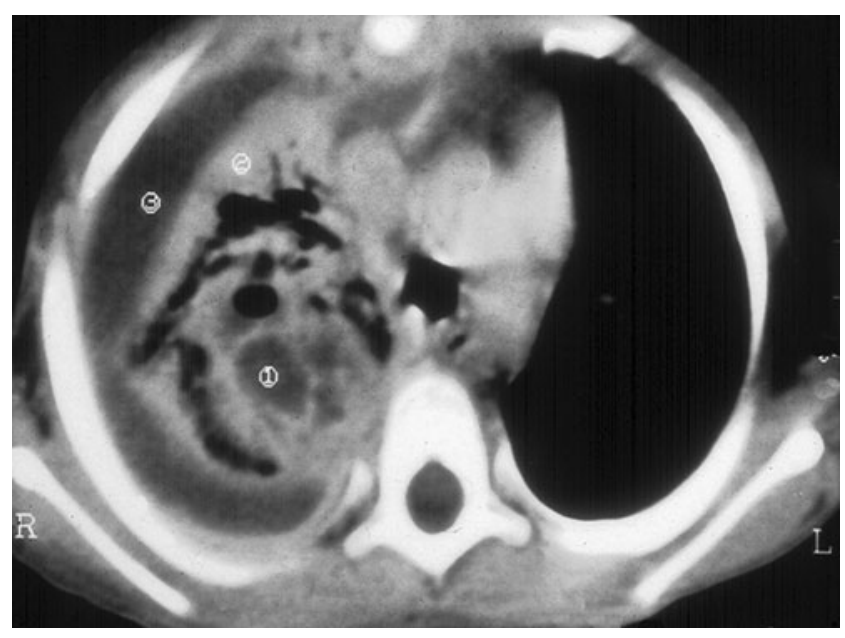

Fig. 21 Disseminated cryptococcosis with necrotizing pneumonia and pleural effusion on axial chest CT. This child also had associated meningoencephalitis

disseminated and miliary presentations of tuberculosis are becoming more frequent among children and even adolescents affected by AIDS (Fig. 22). The main reason is that M. tuberculosis, like HIV infection, also decreases cellular immunity. Mycobacterium avium-intracellulare complex is also encountered in children with AIDS. Imaging findings are nonspecific and similar to those of other forms of mycobacterial infections [2, 41].

Pulmonary infection is also the most common cause of death after bone marrow transplantation. After engraftment (during the first 30 days) profound neutropenia and mucosal injury lead to severe immune suppression. Children at this time are very susceptible to all organisms fungal, bacterial and viral. As the child's neutropenia recovers the immune suppression related to antirejection medication leads to impairment of humoral and cellular immunity (days 30 to 100). These children have a high incidence of cytomegalovirus and RSV. After 100 days the children have near normal immune function and pneumonia is less common. Fungal organisms are associated with very high mortality, with Aspergillus and Candida the most common. It should be noted that children might not demonstrate fever or an elevated white blood cell count. $\mathrm{CT}$ is frequently used to monitor these children when there is a high index of suspicion for disease. Fungal infections in particular demonstrate small, ill-defined nodules with or without surrounding ground-glass opacity. Bacterial infections have features similar to those found in the immunecompetent child [44].

\section{Conclusion}

The role of the radiologist is to help recognize the presence of pneumonia and help guide therapy. The identification of a specific organism is almost never possible from imaging alone. However, by combining imaging and clinical information, the differential diagnoses can be generated. Chest radiographs remain the mainstay of pneumonia imaging, supplemented with sonography and CT for specific indications. Investigation of specific organisms using image-guided sampling and serological determinations is important in designing therapy. During treatment the radiologist can recognize complications such as cavitation, abscess and empyema. The role of image-guided intervention is beyond the scope of this paper, but in many centers the radiologist might be asked to address each of these complications.
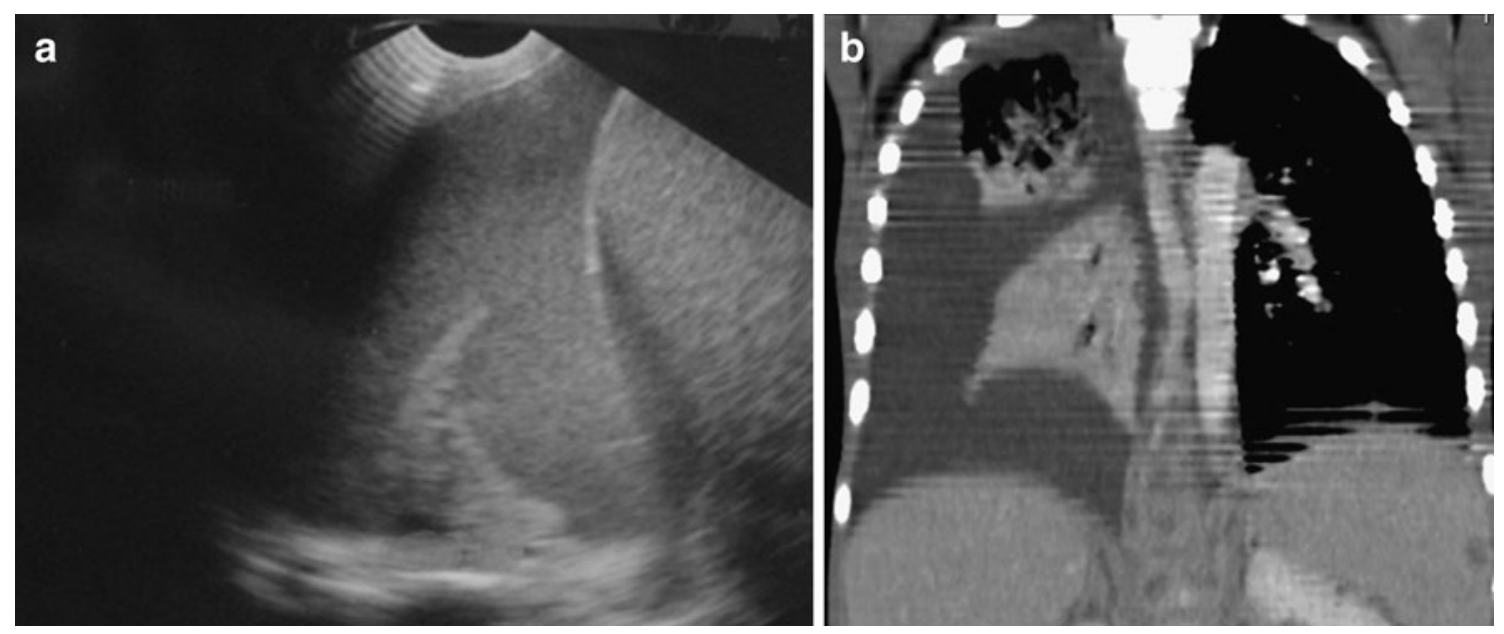

Fig. 22 Tuberculosis in a HAART-naïve, HIV-positive 4-year-old girl. a US and (b) coronal MPR CT images show right-side chylothorax caused by erosion of the thoracic duct by several adjacent thoracic-abdominal lymph nodes with intense peripheral enhancement 


\section{References}

1. Lucaya J, Strife J (2002) Pediatric chest imaging: chest imaging in infants and children, 3rd edn. Springer-Verlag, Berlin

2. Adler B, Effmann E (2008) Pneumonia and pulmonary infection, Chapter 75. In: Slovis TL (ed) Caffey's pediatric diagnostic imaging, 11th edn. Mosby, Philadelphia, pp 1184-1228

3. Marks MJ, Haney PJ, McDermott MP et al (1996) Thoracic disease in children with AIDS. Radiographics 16:1349-1362

4. Swischuk LE, Hayden CK Jr (1986) Viral vs. bacterial pulmonary infections in children (is roentgenographic differentiation possible?). Pediatr Radiol 16:278-284

5. Hollingsworth $\mathrm{C}$ (2005) Thoracic disorders in the immunocompromised child. Radiol Clin North Am 43:435-447

6. Bettenay FA, de Campo JF, McCrossin DB (1988) Differentiating bacterial from viral pneumonias in children. Pediatr Radiol 18:453-454

7. Hamelin ME, Boivin G (2005) Human metapneumovirus: a ubiquitous and long-standing respiratory pathogen. Pediatr Infect Dis J 24(11 Suppl):S203-207

8. Fischer GB, Sarria EE, Mattiello R et al (2010) Post infectious bronchiolitis obliterans in children. Paediatr Respir Rev 11:233-239

9. Virkki R, Juven T, Mertsola J et al (2005) Radiographic follow-up of pneumonia in children. Pediatr Pulmonol 40:223-227

10. Waitches GM, Stern EJ (2002) High-resolution CT of peripheral airway diseases. Radiol Clin North Am 40:21-29

11. Chiu CY, Wong KS, Huang YC et al (2008) Bronchiolitis obliterans in children: clinical presentation, therapy and longterm follow-up. J Paediatr Child Health 44:129-133

12. John SD, Ramanathan J, Swischuk LE (2001) Spectrum of clinical and radiographic findings in pediatric mycoplasma pneumonia. Radiographics 21:121-131

13. Lee I, Kim TS, Yoon HK (2006) Mycoplasma pneumoniae pneumonia: CT features in 16 patients. Eur Radiol 16:719-725

14. Kim YW, Donnelly LF (2007) Round pneumonia: imaging findings in a large series of children. Pediatr Radiol 37:1235-1240

15. Donnelly LF, Klosterman LA (1998) Cavitary necrosis complicating pneumonia in children: sequential findings on chest radiography. AJR 171:253-256

16. Donnelly LF, Klosterman LA (1998) The yield of CT of children who have complicated pneumonia and noncontributory chest radiography. AJR 170:1627-1631

17. Calder A, Owens CM (2009) Imaging of parapneumonic pleural effusions and empyema in children. Pediatr Radiol 39:527-537

18. Starke JR (1999) Tuberculosis in children: where do we go now? Pediatr Pulmonol Suppl 18:140

19. Andronikou S, Wieselthaler N (2004) Modern imaging of tuberculosis in children: thoracic, central nervous system and abdominal tuberculosis. Pediatr Radiol 34:861-875

20. Griffith-Richards SB, Goussard P, Andronikou S et al (2007) Cavitating pulmonary tuberculosis in children: correlating radiology with pathogenesis. Pediatr Radiol 37:798-804

21. Webb R, Muller N, Naidich D (1996) High resolution CT of the lung, 2nd edn. Lippincott-Raven, Philadelphia

22. Sablan B (2009) An update on primary care management for tuberculosis in children. Curr Opin Pediatr 21:801-804

23. Andronikou S (2009) HIV in children: take a moment to make a difference! Pediatr Radiol 39:525-526
24. George R, Andronikou S, Theron S et al (2009) Pulmonary infections in HIV-positive children. Pediatr Radiol 39:545-554

25. Aidé MA (2009) Chapter 4 histoplasmosis. J Bras Pneumol $35: 1145-1151$

26. Kirchner SG, Hernanz-Schulman M, Stein SM et al (1991) Imaging of pediatric mediastinal histoplasmosis. Radiographics $11: 365-381$

27. Khanna G, Kao SC, Kirby P et al (2005) Imaging of chronic granulomatous disease in children. Radiographics 25:11831195

28. Franquet T, Muller NL, Giménez A et al (2001) Spectrum of pulmonary aspergillosis: histologic, clinical, and radiologic findings. Radiographics 21:825-837

29. Thomas KE, Owens CM, Veys PA et al (2003) The radiological spectrum of invasive aspergillosis in children: a 10 -year review. Pediatr Radiol 33:453-460

30. Thia LP, Balfour Lynn IM (2009) Diagnosing allergic bronchopulmonary aspergillosis in children with cystic fibrosis. Paediatr Respir Rev 10:37-42

31. Gasparetto EL, Souza CA, Tazoniero P et al (2007) Angioinvasive pulmonary aspergillosis after allogeneic bone marrow transplantation: clinical and high-resolution computed tomography findings in 12 cases. Braz J Infect Dis 11:110-113

32. Erdem CZ, Erdem LO (2003) Radiological characteristics of pulmonary hydatid disease in children: less common radiological appearances. Eur J Radiol 45:123-128

33. Zi EY, Frush DP, Donnelly LF et al (2001) Primary immunodeficiency disorders in pediatric patients: clinical features and imaging findings. AJR 176:1541-1552

34. Rosen FS, Cooper MD, Wedgwood RJ (1995) The primary immunodeficiencies. N Engl J Med 333:431-440

35. Theron S, Andronkou S, George R et al (2009) Non-infective pulmonary disease in HIV-positive children. Pediatr Radiol 39:555-564

36. Towbin AJ, Chaves I (2010) Chronic granulomatous disease. Pediatr Radiol 40:657-658

37. Merten DF, Buckley RH, Pratt PC et al (1979) Hyperimmunoglobulinemia E syndrome: radiographic observations. Radiology 132:71-78

38. Fitch SJ, Magill HL, Herrod HG et al (1986) Hyperimmunoglobulinemia E syndrome: pulmonary imaging considerations. Pediatr Radiol 16:285-288

39. Manson DE, Sikka S, Reid B et al (2000) Primary immunodeficiencies: a pictorial immunology primer for radiologists. Pediatr Radiol 30:501-510

40. Jeanes AC, Owens CM (2002) Chest imaging in the immunocompromised child. Paediatr Respir Rev 3:59-69

41. Marchiori E, Müller NL, Soares Souza A Jr et al (2005) Pulmonary disease in patients with AIDS: high-resolution CT and pathologic findings. AJR 184:757-764

42. Severo CB, Xavier MO, Gazzoni AF et al (2009) Cryptococcosis in children. Paediatr Respir Rev 10:166-171

43. Berman DM, Mafut D, Djokic B et al (2007) Risk factors for the development of bronchiectasis in HIV-infected children. Pediatr Pulmonol 42:871-875

44. Escuissato DL, Gasparetto EL, Marchiori E et al (2005) Pulmonary infections after bone marrow transplantation: high-resolution CT findings in 111 patients. AJR 185:608615 